\title{
The Association between Attachment Avoidance and Quality of Life in Bariatric Surgery Candidates
}

\author{
Sanjeev Sockalingam ${ }^{\mathrm{a}}$ Susan Wnuk ${ }^{\mathrm{a}}$ Rachel Strimas $^{\mathrm{a}}$ Raed Hawa $^{\mathrm{a}}$ Allan Okrainec ${ }^{\mathrm{b}}$ \\ ${ }^{\text {a }}$ Department of Psychiatry, \\ ${ }^{\mathrm{b}}$ Division of Surgery, University Health Network, University of Toronto, Toronto, ON, Canada
}

\section{Keywords}

Bariatric surgery · Mood · Psychological aspects . Depression · Quality of life · Relationship style

\section{Summary}

Objective: Patients presenting for bariatric surgery have high rates of psychiatric co-morbidity and reduced health-related quality of life (HROOL) compared to the general population. In this study, we aimed to determine the relationship between insecure attachment styles and HRQOL in bariatric surgery candidates. Methods: We assessed depression, social support, attachment avoidance, attachment anxiety, HROOL (SF-36), and eating disorder psychopathology in 70 consecutive patients assessed for bariatric surgery. SF-36 physical (PCS) and mental component scores (MCS) were compared to a normative sample and analyzed using t-tests. Predictors of HROOL were analyzed using multiple linear regression analyses. Results: SF-36 PCS and MCS in this prebariatric surgery sample were significantly lower than in an age-matched reference population. Depression, attachment anxiety, attachment avoidance and eating disorder psychopathology scores were negatively correlated with SF-36 MCS. Depression was associated with lower SF-36 PCS ( $p=0.015$ ). SF-36 MCS were significantly predicted by BDI scores $(p<0.001)$ and attachment avoidance $(p=0.024)$ in our multiple regression model. Conclusion: This is the first study to demonstrate an association between attachment avoidance and poor mental HRQOL in bariatric surgery candidates. Future studies are needed to examine the effect of attachment avoidance on post-bariatric surgery outcomes.

\section{Introduction}

Although bariatric surgery has demonstrated its efficacy in achieving substantial weight loss in most patients and subsequent improvement in obesity-related morbidity, improvement in health-related quality of life (HRQOL) is an equally important outcome. Several psychological variables related to HRQOL, including attachment style, depression, and social support as well as the relationships between these variables are examined in the present study.

HRQOL refers to patients' self-perceived multidimensional health status and encapsulates physical status, symptom status, functional status, health perceptions, and general wellbeing [1]. Body weight alone has been shown to negatively impact quality of life [2]. In a mixed obese sample including 153 bariatric surgery patients, de Zwaan et al. [3] showed a significant dose-response association between BMI predicting worse physical health impairment, which is consistent with previously established findings [4]. However, BMI was not a significant predictor of the mental component of quality of life in this specific study.

Data supports an improvement in HRQOL in a majority of obese patients receiving bariatric surgery in comparison to untreated severely obese patients [5, 6]. In a review of 26 studies representing 2,010 patients and five different surgical procedures, it was found that surgery resulted in improvements in quality of life along with weight loss [7]. However, it is important to understand why some post-operative patients do not experience improvement in HRQOL with this intervention and whether differences in HRQOL in pre-surgery patients can provide some insight into this question.

Several studies have examined the impact of psychosocial factors on HRQOL in obese patient populations. Psychiatric co-morbidity has been associated with decreased HRQOL, in both physical and mental domains, in obese patient samples

\section{KARGER \\ Fax +497614520714 \\ Information@Karger.de}

www.karger.com (c) 2011 S. Karger GmbH, Freiburg

1662-4025/11/0046-0456\$38.00/0

Accessible online at:

www.karger.com/ofa
Dr. Sanjeev Sockalingam

Department of Psychiatry, University Health Network

University of Toronto, 200 Elizabeth Street - 8EB-228, Toronto, ON, M5G2C4, Canada

sanjeev.sockalingam@uhn.on.ca 
[3]. Moreover, eating psychopathology, such as grazing eating patterns, binge eating and loss of control over eating, have also been associated with reduced HRQOL, especially in mental health-related QOL domains [8-10]. However, less is known about the association between relationship or attachment styles and HRQOL in bariatric surgery samples.

Attachment theory, founded by John Bowlby [11], provides a framework for understanding one's experience of chronic illnesses, and attachment style has been associated with obesity $(11,12)$. Bowlby [11] purported that individuals' ability to reach out for social support during an illness event is based on relationship patterns that develop during one's childhood and remain relatively stable throughout adulthood. Adult attachment styles can be categorized into secure attachment and two insecure attachment styles, namely, avoidant and anxious attachment [13]. Individuals with an anxious attachment fear interpersonal rejection or abandonment have an excessive need for approval from others and experience intense distress when relationship partners are unavailable or unresponsive. Individuals with an avoidant/dismissing style fear dependence and interpersonal intimacy have an excessive need for self-reliance and independence and are reluctant to self-disclose.

The salience of attachment style in obesity is supported by research examining the role of attachment style on eating disorder symptoms. In addition, research has shown a relationship between insecure attachment and obesity, with higher BMI being associated with anxious attachment [12]. Given the stability of attachment style in comparison to other psychosocial variables, insecure attachment style may provide valuable insights into the development of obesity and response to weight loss interventions, such as bariatric surgery, and HRQOL. However, D'Argenio et al. [14] failed to demonstrate a robust effect between attachment style and obesity, and highlighted the need for further exploration.

In addition to the contribution of attachment style to the development of obesity, poor attachment patterns may lead to reduced HRQOL and increased psychopathology in bariatric surgery candidates. Eating psychopathology has been linked to insecure attachment style [15], and studies involving individuals with disordered eating have demonstrated higher rates of attachment insecurity [16]. Further research is needed to clearly elucidate the relationship between attachment style and eating psychopathology in bariatric surgery candidates.

If attachment insecurity predisposes an individual to greater eating psychopathology and increased BMI, it is likely that insecure attachment style will also lead to reduced HRQOL. Research also suggests that greater social support may mitigate the role of obesity on HRQOL in male, but not female, obese patients [17]. Due to the paucity of literature, the influence of social support and insecure attachment styles, namely anxious or avoidant styles, on HRQOL in bariatric surgery candidates remains unknown.
Given the potential implications of attachment style on bariatric surgery patient psychopathology and HRQOL, the purpose of this study was to examine the attachment styles of bariatric surgery candidates presenting for pre-surgery assessment. We hypothesized that an avoidant attachment style would be associated with lower HRQOL given its lack of responsiveness to social support in comparison to anxious attachment [18].

\section{Material and Methods}

\section{Study Sample}

70 consecutively referred adult bariatric surgery candidates assessed by the Toronto Western Hospital (TWH) Bariatric Surgery Program in Toronto, ON, Canada participated in the study. The TWH Bariatric Surgery Program is one of two bariatric surgery assessment centers within the five-hospital University of Toronto Bariatric Surgery Collaborative. The mandate of the Bariatric Surgery Collaborative is to perform the laparoscopic Roux-en-Y gastric bypass procedure. To be eligible for surgery, patients must have a BMI $\geq 40$ or $\geq 35 \mathrm{~kg} / \mathrm{m}^{2}$ with a co-morbid obesity-related condition such as obstructive sleep apnea, hypertension, or diabetes mellitus. This study was approved by the institutional Research Ethics Board at the University Health Network in Toronto and conformed to the principles outlined in the Declaration of Helsinki.

\section{Procedure}

All patients referred to the TWH Bariatric Surgery Program received a comprehensive pre-surgery interdisciplinary assessment. The assessment process involved interviews with a nurse, social worker, dietician, and a psychologist, psychometrist (an individual with expertise in psychodiagnostic testing) or psychiatrist. Patients completed a minimum of six interviews as part of the pre-surgery assessment process, a self-report questionnaire package, and two educational group sessions focusing on the Roux-en-Y gastric bypass procedure and nutrition. Surgery candidates who chose to proceed with bariatric surgery were included in the present analysis.

\section{Psychosocial Measures}

Patients completed the Beck Depression Inventory (BDI) a self-report measure of symptoms of depression [19]. The BDI is a 21-item scale and has been used already in bariatric surgery patient populations [20]. Individual items are rated from 0 to 3 to indicate severity and are summed to create a total score. Higher scores demonstrate greater severity of depressive symptoms. Cut-off scores have been established to indicate severity: 0-9 indicates minimal depression, 10-18 indicates mild depression, 19-29 indicates moderate depression, and 30-63 indicates severe depression.

HRQOL was evaluated using the SF-36, a widely used HRQOL measure [21]. Its use in bariatric surgery patient populations is well-established [22]. The SF-36 is composed of eight dimensions that assess physical and mental health. Physical functioning, physical role functioning, bodily pain, and general health form the physical component score (PCS) and vitality, social functioning, emotional role functioning, and mental health form the mental component score (MCS). The PCS and MCS were used as outcome variables in the present study.

Attachment style was assessed using the Experiences in Close Relationships scale (ECR-16), a 16-item scale that has been validated against the longer ECR-32 [23]. The ECR-16 yields a total anxious and avoidant attachment score based upon scoring of 8 items to each attachment style. Each attachment score ranges from 8 to 56 with higher anxious or avoidant attachment scores representing greater attachment security. 
Table 1. Patient characteristics $(n=70)$

\begin{tabular}{ll}
\hline Female gender, n $(\%)$ & $64(90.1 \%)$ \\
Age, years & $44.26 \pm 9.9$ \\
BMI, kg/m² & $47.08 \pm 6.1$ \\
BDI & $13.26 \pm 9.2$ \\
EDE-Q total score & $3.10 \pm 1.0$ \\
Attachment anxiety score & $26.3 \pm 9.9$ \\
Attachment avoidance score & $24.87 \pm 10.9$ \\
Medical Outcomes Study Social Support Survey & $85.71 \pm 15.9$ \\
SF-36 PCS & $31.04 \pm 9.6$ \\
SF-36 MCS & $46.43 \pm 11.6$ \\
\hline
\end{tabular}

Social support was measured with the Medical Outcomes Study Social Support Survey (MOS-SSS), a valid and reliable self-report questionnaire for measuring social support [24]. It consists of four social support subscales and provides an overall social support index. Higher scores on the MOS-SSS represent more support. The MOS-SSS has been previously used in bariatric surgery patients [25].

Eating pathology was measured using the Eating Disorder Examination-Questionnaire (EDE-Q), a 32-item self-report questionnaire based upon the structured eating disorder interview, the Eating Disorder Examination [26]. The EDE-Q consists of four subscales: dietary restraint, eating concern, weight concern, and shape concern. Items are rated on a 7-point scale. The EDE-Q has demonstrated good validity and reliability in measuring eating-related pathology and behaviors and has been used in bariatric surgery patients [27, 28].

Finally, the height and weight of patients were measured by a clinic dietician, and the BMI of the patients was calculated.

\section{Statistical Analysis}

All statistical analyses were conducted using PASW 18.0. Continuous variables are reported as means \pm standard deviations (SD), and categorical variables are reported in percentages and number of patients $(n)$. The mean scores on the SF-36 PCS and MCS were compared to age-matched Canadian general population norms using student t-tests [29]. Pearson's correlation coefficients were used to determine statistically significant associations with each psychosocial variable and the SF-36 PCS and MCS.

A multiple linear regression analysis was performed to determine the strength of each predictor of physical and mental quality of life as per the SF-36. Assumptions of multiple linear regression were met before proceeding with the analysis. We entered BDI scores, EDE-Q scores, MOSSSS scores, attachment anxiety, attachment avoidance, BMI, and age as predictors of our model. Statistical significance was defined as a $\mathrm{p}<0.05$ for all data.

\section{Results}

\section{Participant Characteristics}

Participant characteristics are summarized in table 1. Our study sample was predominantly female $(90 \%)$, with a mean BMI of $47.1 \pm 6.1 \mathrm{~kg} / \mathrm{m}^{2}$ and a mean age of $44.3 \pm 9.9$ years The mean BDI score was $13.26 \pm 9.2$, indicating mild severity of depressive symptoms. SF-36 PCS (31.04 \pm 9.62$)$ and MCS $(46.43 \pm 11.60)$ were significantly lower than age-matched SF-36 PCS $(52.0 \pm 8.0)(\mathrm{t}(565)=19.75, \mathrm{p}<0.001)$ and MCS $(50.9 \pm 9.0)(\mathrm{t}(565)=3.70, \mathrm{p}<0001)$, indicating poorer physical and mental health quality of life in bariatric surgery patients relative to the Canadian general population.
Table 2. Regression analysis for SF-36 PCS and MCS

\begin{tabular}{|c|c|c|c|c|}
\hline \multirow[t]{2}{*}{ Predictor variables } & \multicolumn{2}{|l|}{ SF-36 PCS } & \multicolumn{2}{|l|}{ SF-36 MCS } \\
\hline & beta & $\mathrm{p}$ value & beta & $\mathrm{p}$ value \\
\hline BDI score & -0.442 & 0.015 & -0.543 & $<0.001$ \\
\hline ECR-Anxious & -0.098 & NS & -0.205 & NS \\
\hline ECR-Avoidant & 0.154 & NS & -0.207 & 0.024 \\
\hline BMI & 0.181 & NS & 0.003 & NS \\
\hline MOS-SSS & -0.238 & NS & -0.091 & NS \\
\hline EDE-Q total score & -0.155 & NS & 0.058 & NS \\
\hline Age & -0.252 & NS & 0.098 & NS \\
\hline F-Statistic (df) & $2.56(7,51)$ & 0.024 & $9.90(7,51)$ & $<0.001$ \\
\hline Adjusted R-square & 0.158 & & 0.518 & \\
\hline
\end{tabular}

NS = Statistically not significant.

\section{Predictors of HRQOL}

Correlation coefficients for SF-36 PCS and MCS and the specific psychosocial variables yielded several significant associations. SF-36 PCS were significantly negatively correlated with BDI scores $(\mathrm{r}=-0.335, \mathrm{p}=0.006)$ and EDE-Q $(\mathrm{r}=-0.259$, $\mathrm{p}=0.041)$. A significant negative correlation was found between SF-36 MCS and the following variables: BDI scores $(\mathrm{r}=-0.757, \mathrm{p}<0.001)$, ECR anxiety $(\mathrm{r}=-0.458, \mathrm{p}<0.001)$, ECR avoidance $(\mathrm{r}=-0.594, \mathrm{p}<0.001)$, and EDE-Q total score $(\mathrm{r}=-0.483, \mathrm{p}<0.001)$. Social support was positively associated with an increase in SF-36 MCS $(r=0.441, \mathrm{p}<0.001)$. Both ECR anxiety $(r=0.475, \mathrm{p}<0.001)$ and ECR avoidance $(\mathrm{r}=$ $0.528, \mathrm{p}<0.001)$ were significantly correlated with BDI scores.

Table 2 summarizes the multiple linear regression analysis results for SF-36 PCS and MCS. The multiple regression analysis predicting PCS was statistically significant $(\mathrm{p}=0.024)$, with greater depressive symptoms predicting lower physical health quality of life $(p=0.015)$. In addition, the model for predictors of MCS was statistically significant $(\mathrm{p}<0.001)$, with greater depressive symptoms $(\mathrm{p}<0.001)$ and greater avoidant attachment style $(\mathrm{p}=0.024)$ predicting worse mental health quality of life.

\section{Discussion}

This study is the first study to explore associations between attachment styles and quality of life in bariatric surgery candidates. An important finding of this study is that attachment avoidance was one of two variables significantly associated with SF-36 mental health scores and highlights the need to consider patients' relationship style when studying mental health quality of life in bariatric surgery candidates.

Our findings confirm a previously established finding that depressive symptoms are more closely associated with HRQOL than BMI in patients with extreme obesity [30]. Although depressive symptoms have been consistently associ- 
ated with reduced mental HRQOL, the effects of depressive symptoms on physical HRQOL are inconclusive [31, 32]. Nonetheless, Ali et al. [33] suggest that depression may be related to increased severity and prevalence of obesity-related co-morbidity independent of BMI and may indirectly impact physical health quality of life. Therefore, this could explain the observed relationship between depression and physical HRQOL in our sample.

The novel association between avoidant attachment and mental HRQOL in bariatric surgery candidates warrants further discussion. In our study, both anxious and avoidant attachment styles were significantly correlated with depressive symptoms, arguing against depression alone as the mediating factor accounting for this difference in mental health quality of life. A possible explanation may be the role played by emotion regulation strategies. In an eating disorder sample, attachment anxiety contributed to both depressive symptoms and eating disorder symptoms through increased emotional reactivity, whereas the relationship between attachment avoidance and depressive symptoms was mediated by emotion deactivation [34]. Furthermore, only attachment avoidance had a direct relationship to eating disorder symptoms. These findings suggest that emotion regulation may be a mediating factor between attachment style and psychopathology.

Furthermore, individuals with avoidant attachment style are less likely to seek social support when distressed, which contrasts the response of individuals with anxious attachments [13]. The limited use of social supports in avoidant attachment style patients could be a contributing factor to increased distress and poor coping capacity in this sub-group of patients.

In addition, evidence suggests that anxious attachment styles may be malleable to social support. Green et al. [18], for instance, found a decrease in anxious attachment in mothers who perceived greater social support in low stress situations and showed no change in high stress situations. In addition, they found avoidant attachment to be insensitive to changes in social support, suggesting that low social support may only impact anxiously attached patients and result in lower mental HRQOL. It is also possible that other explanations may be responsible for this result, including the types and sensitivity of measures used and insufficient statistical power.

These results should be interpreted in the context of study limitations. Our study involved 70 patients; a larger sample size could detect additional predictors and may explain our inability to identify significant predictor variables for physically related quality of life domains in our model. The present study utilized self-report measures and could be enhanced with the use of rigorous structured clinical interviews to determine the relationship between specific psychiatric disorders, attachment style and HRQOL. Furthermore, this is a crosssectional study; large prospective, controlled studies are needed to evaluate the predictive value of depression and avoidant attachment style on HRQOL and weight loss following bariatric surgery.

In summary, our study identifies patient relationship style as a major factor in determining HRQOL in bariatric surgery patients primarily in the mental health domains. Moreover, depressive symptoms were associated with significant reductions in physical and mental HRQOL in this patient population. Despite literature suggesting a role for social support in predicting HRQOL, our study argues for greater consideration of bariatric surgery patients' attachment style and depression when considering quality of life outcomes in bariatric surgery candidates. Future studies are needed to examine if specific relationship styles, namely avoidant attachment style, can predict weight change, diet adherence, compliance with follow-up appointments, and mental health outcomes post surgery.

\section{Disclosure Statement}

The authors declared no conflict of interest.

\section{References}

1 Wilson IB, Cleary PD: Linking clinical variables with health-related quality of life. A conceptual model of patient outcomes. JAMA 1995;273:59-65

2 Yancy WS, Jr., Olsen MK, Westman EC, Bosworth HB, Edelman D: Relationship between obesity and health-related quality of life in men. Obes Res 2002;10:1057-1064.

3 de Zwaan M, Petersen I, Kaerber M, Burgmer R, Nolting B, Legenbauer T, et al: Obesity and quality of life: a controlled study of normal-weight and obese individuals. Psychosomatics 2009;50:474-482.

4 Doll HA, Petersen SE, Stewart-Brown SL: Obesity and physical and emotional well-being: associations between body mass index, chronic illness, and the physical and mental components of the SF-36 questionnaire. Obes Res 2000;8:160-170.
5 Adams TD, Pendleton RC, Strong MB, Kolotkin RL, Walker JM, Litwin SE, et al: Health outcomes of gastric bypass patients compared to nonsurgical, nonintervened severelu obese. Obesity 2010;18:121-130.

6 Hernandez LV, Klyve D: Quality-adjusted life expectancy benefits of laparoscopic bariatric surgery: a United States perspective. In J Technol Assess 2010;26:280-287.

7 Jayasekara R: Weight loss surgery for obesity. Am J Nurs 2010;110:61.

8 Colles SL, Dixon JB, O'Brien PE: Loss of control is central to psychological disturbance associated with binge eating disorder. Obesity 2008;16:608-614.

$\checkmark 9$ De Zwaan M, Mitchell JE, Howell LM, Monson N, Swan-Kremeier L, Roerig JL, et al: Two measures of health-related quality of life in morbid obesity. Obes Res 2002;10:1143-1151.
10 Kofman MD, Lent MR, Swencionis C: Maladaptive eating patterns, quality of life, and weight outcomes following gastric bypass: results of an internet survey. Obesity 2010;18:1938-1943.

11 Bowlby J: Attachment and Loss, vol. 1: Attachment. New York, Basic Books, 1969.

12 Hintsanen M, Jokela M, Pulkki-Raback L, Viikari JS, Keltikangas-Jarvinen L: Associations of youth and adulthood body-mass index and waist-hip ratio with attachment styles and dimensions. Curr Psychol 2010;29:257-271.

13 Mikulincer M, Shaver PR, Pereg D: Attachment theory and affect regulation: the dynamics, development, and cognitive consequences of attachmentrelated strategies. Motiv Emot 2003;27:77-102. 
14 D’Argenio A, Mazzi C, Pecchioli L, Di Lorenzo G, Siracusano A, Troisi A: Early trauma and adult obesity: is psychological dysfunction the mediating mechanism? Physiol Behav 2009;98:543-546.

15 Troisi A, Massoroni P, Cuzzolaro M: Early separation anxiety and adult attachment style in women with eating disorders. Br J Clin Psychol 2005;44:89-97.

16 Ward A, Ramsay R, Treasure J: Attachment research in eating disorders. Br J Med Psychol 2000; 73:35-51.

17 Wiczinski E, Doring A, John J, von Lengerke T: Obesity and health-related quality of life: does social support moderate existing associations? Br J Clin Psychol 2009;14:717-734.

18 Green BL, Furrer CJ, McAllister CL: Does attachment style influence social support or the other way around? A longitudinal study of Early Head Start mothers. Attach Hum Dev 2011;13:27-47.

19 Beck AT, Bearnesderfer A: Assessment of depression: the depression inventory. Mod Probl Pharmacopsychiatry 1974;7:151-169.

20 Krukowski RA, Friedman KE, Applegate KL. The utility of the Beck Depression Inventory in a bariatric surgery population. Obes Surg 2010;20:426-431.

21 Ware JE, Snow KK, Kosinski M, Gandek B: SF-36 Health Survey Manual and Interpretation Guide. Boston, The Health Institute, New England Medical Center; 1993.
22 de Zwaan M, Lancaster KL, Mitchell JE, Howell LM, Monson N, Roehrig JL, et al: Health-related quality of life in morbidly obese patients: effect of gastric bypass surgery. Obes Surg 2002;12:773-780.

23 Brennan KA, Clark CL, Shaver PR. Self-report measurement of adult attachment; in Simpson JA, Rhodes WS (eds): Attachment Theory and Close Relationships. New York, Guildford, 1988. pp 47-76.

24 Sherbourne CD, Stewart AL: The MOS social support survey. Soc Sci Med. 1991;32:705-714.

25 Livihits M, Mercado C, Yermilov I, Parikh JA, Dutson E, Mehran A, et al: Is social support associated with greater weight loss after bariatric surgery? A systematic review. Obes Rev 2011;12:142-148.

26 Black CM, Wilson GT: Assessment of eating disorders: interview versus questionnaire. Int $\mathrm{J}$ Eat Disord 1996;20:43-50.

27 Grilo CM, Masheb RM, Wilson GT. Different methods for assessing the features of eating disorders in patients with binge eating disorder: a replication. Obes Res 2001;9:418-422.

28. Kalarchian MA, Marcus MD, Wilson GT, Labouvie EW, Brolin RE, LaMarca LB: Binge eating among gastric bypass patients at long-term followup. Obes Surg 2002;12:270-275.
29 Hopman WM, Towheed T, Anastassiades T, Tenenhouse A, Poliquin S, Berger C, et al: Canadian normative data for the SF-36 health survey. CMAJ 2000;163:265-271.

30 Fabricatore AN, Wadden TA, Sarwer DB, Faith MS: Health-related quality of life and symptoms of depression in extremely obese persons seeking bariatric surgery. Obes Surg 2005;15:304-309.

31 Andersen JR, Aasprang A, Bergsholm P, Sletteskog N, Vage V, Natvig GK: Predictors for health-related quality of life in patients accepted for bariatric surgery. Surg Obes Relat Dis 2009;5:329-333.

32 Sanchez-Santos R, Del Barrio MJ, Gonzalez C, Madico C, Terrado I, Gordillo ML, et al: Longterm health-related quality of life following gastric bypass: influence of depression. Obes Surg 2006;16: 580-585.

33 Ali MR, Rasmussen JJ, Monash JB, Fuller WD: Depression is associated with increased severity of co-morbidities in bariatric surgical candidates. Surg Obes Relat Dis 2009;5:559-564.

34 Tasca GA, Szadkowski L, Illing V, Trinneer A, Grenon R, Demidenko N, et al: Adult attachment, depression, and eating disorder symptoms: the mediating role of affect regulation strategies. Pers Individ Dif 2009;47:662-667. 